\title{
The effect of different doses of chloroprocaine on saddle anesthesia in perianal surgery ${ }^{1}$
}

\author{
Ying ZhangI, Yang Bao" ${ }^{\mathrm{II}}$, Linggeng $\mathrm{Li}^{\mathrm{III}}$, Dongping Shi ${ }^{\mathrm{IV}}$ \\ 'Master, Department of Anesthesiology, Shanghai Jiading Central Hospital, Shanghai, China. Conception and design of the study; acquisition, analysis \\ and interpretation of data; manuscript writing, final approval.

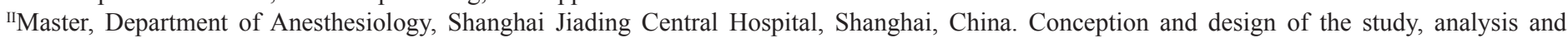 \\ interpretation of data, critical revision. \\ IIIMaster, Department of Anesthesiology, Shanghai Jiading Central Hospital, Shanghai, China. Histopathological examinations, critical revision.

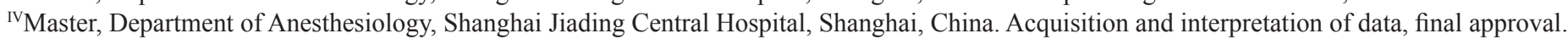

\section{ABSTRACT}

PURPOSE: To investigate a saddle anesthesia with different doses of chloroprocaine in perianal surgery.

METHODS: Total 60 Patients aged 18-75 years (Anesthesiologists grade I or II) scheduled to receive perianal surgery. Patients using saddle anesthesia were randomized to group A, group B and group $\mathrm{C}$ with the same concentration $(0.5 \%)$ chloroprocaine with different doses $1.0 \mathrm{~mL}, 0.8 \mathrm{~mL}$ and $0.6 \mathrm{~mL}$, respectively. Systolic blood pressure (SBP), diastolic blood pressure (DBP), heart rate (HR) and the sensory and motor block were recorded to evaluate the anesthesia effect of chloroprocaine in each group.

RESULTS: The duration of sensory block of group $\mathrm{C}$ is shorter than those of group A and B. The maximum degree of motor block is observed (group C: 0 level, group A: III level; and group B: I level) after 15 minutes. Besides, there was a better anesthetic effect in group B than group A and group C, such as walking after saddle anesthesia. However, there is also no significant difference of blood pressure decreasing in these three groups.

CONCLUSION: It's worth to employ a saddle anesthesia with appropriate doses of chloroprocaine in clinical perianal surgery. Key words: Anesthesia, Local. Urinary Retention. 


\section{Introduction}

The optimal anesthetic technique would provide for excellent operating conditions, a rapid recovery, nopostoperative side effects, and high patient satisfaction. In addition to increasing the quality and decreasing the costs of the anesthetic services, the ideal anesthetic technique would also improve operating room (OR) efficiency and provide for an early discharge. Saddle anesthesia is effective in patients experiencing perianal surgery in terms of analgesic consumption within 24 hours after surgery and aspects of postoperative recovery in view of all aspects of the anesthesia technique ${ }^{1-3}$.

Chloroprocaine ( $\mathrm{pKa} 8.7$ ) is the drug of choice for epidural analgesia and a decompensating fetus, because it does not participate in ion trapping. Placental transfer of 2-chloroprocaine is not influenced by fetal acidosis ${ }^{4,5}$. Chloroprocaine (trade name Nesacaine or Nesacaine-MPF) is a local anesthetic given by injection during surgical procedures and labor and delivery. Chloroprocaine constricts blood vessels resulting in reduced blood loss; this is in contrast to other local anesthetics e.g. lidocaine, which do not do such. Chloroprocaine is an ester anesthetic (Figure 1). Chloroprocaine was developed to meet the need for a short acting spinal anaesthetic that is reliable and has a favorable safety profile to support the growing need for day case surgery ${ }^{6-8}$.

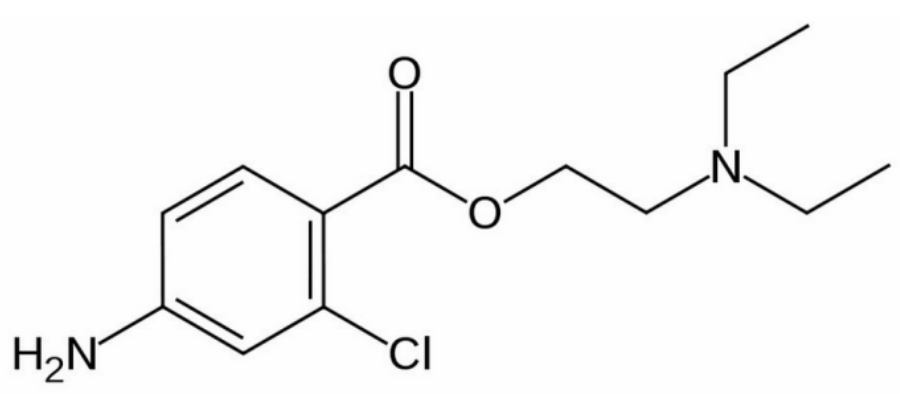

FIGURE 1 - Chemical structure of chloroprocaine.

Chloroprocaine dose will usually begin with $11 \mathrm{mg} /$ $\mathrm{kg} /$ dose up to $800 \mathrm{mg}$ depending in the indication though can change because it may be based upon the relative severity of the underlying problem and any comfort level the physician treating a particular patient has with both the medication and the disease process ${ }^{9}$. For almost all pharmaceuticals often the higher a dosage more commonly a given patient will experience medicine side effects ${ }^{10}$.

The purpose of this paper was to investigate a saddle anesthesia with different doses of chloroprocaine at the same concentration $(0.5 \%, \mathrm{w} / \mathrm{v})$ in terms of systolic blood pressure
(SBP), diastolic blood pressure (DBP), heart rate (HR), sensory nerve, motor nerve block situations, side effects of intraoperative and postoperative anesthetic method.

\section{Methods}

All persons have given their informed consent prior to their inclusion in the study, and all human studies have been approved by according Ethics Committee and performed in accordance with the ethical standards. Between September 2011 and December 2012, 60 patients aged 18-75 years with American Society of Anesthesiologists (ASA) grade I or II undergoing perianal surgery were eligible for the study. The patients were randomly divided into three groups: (A) $0.5 \%$ $(\mathrm{w} / \mathrm{v})$ chloroprocaine dissolved in $1.0 \mathrm{~mL} 10 \%(\mathrm{w} / \mathrm{v})$ glucose solution; (B) $0.5 \%(\mathrm{w} / \mathrm{v})$ chloroprocaine dissolved in 0.8 $\mathrm{mL} 10 \%(\mathrm{w} / \mathrm{v})$ glucose solution; (C) $0.5 \%$ (w/v) chloroprocaine dissolved in $0.6 \mathrm{~mL} 10 \%(\mathrm{w} / \mathrm{v})$ glucose solution, each group includes 20 cases.

\section{Saddle anesthesia method}

Saddle anesthesia was performed under aseptic conditions, using a standard midline approach in the sitting position. Anesthetic in each group was injected into the subarachnoid space at the L4-L5 interspace, respectively. Before the anesthetists tested for sensory anesthesia, all patients should be remained in the sitting position for $10 \mathrm{~min}$. The pinching was applied to the anal orifice with a long surgical clamp, then moved in different diagonal directions until satisfactory anesthesia had reached. Patients were recorded systolic blood pressure (SBP), diastolic blood pressure (DBP) and heart rate (HR) in calm sitting in burglary $\left(\mathrm{T}_{1}\right)$, injecting anesthetic into waist $\left(\mathrm{T}_{2}\right), 5 \min \left(\mathrm{T}_{3}\right), 10 \min \left(\mathrm{T}_{4}\right), 15 \min \left(\mathrm{T}_{5}\right)$ and collapse $\left(\mathrm{T}_{6}\right)$, respectively. Patients with a sufficient saddle anesthesia were transferred to the operating theatre. Patients were observed the incidence of uroschesis, blood pressure decreasing, headache and sore throat.

After injection, sensory and motor block were observed and recorded in each group. The start time of sensory block is painless acupuncture saddle area, and the start time of motor block is the lifting the lower limb weakness after injection. Patients in each group were measured degree of motor block at five, ten and 15 min after injection (adopt modified Bromage to rank lower limb motor block: (0) no motor block; (I) cannot bend the hip; (II) cannot bend knee joint; (III) cannot bend the ankle) ${ }^{11}$. 


\section{Statistical analysis}

The SPSS software (IBM, USA) was used for statistical purposes. Quantitative data are presented as mean (s.d.) values or as median (range).

\section{Results}

In this study, 60 patients underwent perianal surgery, treated with saddle anesthesia with different doses of chloroprocaine at the same concentration $(0.5 \%, \mathrm{w} / \mathrm{v})$. Demographic data of these 60 patients who receiving saddle anesthesia were revealed in Table 1.There is no significant difference of patient age, gender and ASA classification in three groups.

TABLE 1 - Demographic data of patients receiving saddle anesthesia.

\section{Character}

Sex ratio(M:F)

Age(years)*

$\operatorname{Height}(\mathrm{cm})^{*}$

Weight $(\mathrm{kg}) *$

\section{Saddle anesthesia $(n=60)$}

$38: 21$

35

172

60.7
The change of SBP, DBP and HR in three groups is revealed in Table 2. There is no significant difference of SBP, DBP or HR between $T_{6}$ and $T_{1}$ in three groups. All three groups of patients achieve satisfactory anesthesia, anal relaxation, perianal nerve block improvement, and weak impact on vital signs.

Sensory and motor block of these three groups are shown in Tables 3 and 4, intraoperative and postoperative adverse reactions and complications are shown in Table 5 (Tables 3, 4 and 5).

There is no significant difference of the block starting time of the sensory and motor block in three groups, but the block duration of sensory was shorter in group $\mathrm{C}(2.1 \pm 0.22 \mathrm{~h})$ than group $\mathrm{A}(5.2 \pm 0.25 \mathrm{~h})$ and $\mathrm{B}(3.5 \pm 0.17 \mathrm{~h})$. Maximum degree of motor block is observed (group C: 0 level; group A: III level; and group B: I level) after 15 minutes.

As shown in Table 5, there is no significant difference of blood pressure decreasing in these three groups. Moreover, there are one case of uroschesis in group C, 6 cases in group A and 4 cases in group B.

*Values are mean (s.d.).

TABLE 2 - The change of SBP, DBP and HR in three groups of patients $( \pm s)$.

\begin{tabular}{cccccccc}
\hline Group & Case number & T1 & T2 & T3 & T4 & T5 \\
\hline A & 20 & & & & & T6 \\
SBP & & $125.6 \pm 8.2$ & $126.0 \pm 10.5$ & $121.4 \pm 13.0$ & $117.0 \pm 10.0$ & $123.0 \pm 8.4$ & $83.8 \pm 11.5^{*}$ \\
DBP & & $83.4 \pm 9.2$ & $80.7 \pm 10.2$ & $80.0 \pm 12.5$ & $78.5 \pm 10.2$ & $81.0 \pm 10.5$ & $47.5 \pm 4.0^{*}$ \\
HR & & $82.5 \pm 10.2$ & $80.5 \pm 11.4$ & $80.5 \pm 11.2$ & $79.4 \pm 10.5$ & $81.0 \pm 11.5$ & $58.5 \pm 2.2^{*}$ \\
B & 20 & & & & & \\
SBP & & $131.4 \pm 10.4$ & $118.2 \pm 16.5$ & $125.8 \pm 8.2$ & $125.5 \pm 11.2$ & $126.0 \pm 12.4$ & $77.5 \pm 14.4^{*}$ \\
DBP & & $88.6 \pm 9.2$ & $78.5 \pm 15.2$ & $85.8 \pm 9.2$ & $87.5 \pm 9.2$ & $84.0 \pm 5.5$ & $46.4 \pm 9.5^{*}$ \\
HR & & $81.2 \pm 10.0$ & $78.2 \pm 14.2$ & $80.2 \pm 13.4$ & $86.0 \pm 11.0$ & $82.8 \pm 11.5$ \\
C & 20 & & & & & $49.3 \pm 5.5^{*}$ \\
SBP & & $124.8 \pm 8.2$ & $125.3 \pm 10.4$ & $121.5 \pm 12.5$ & $124.2 \pm 12.8$ & $126.9 \pm 12.5$ & $77.6 \pm 14.7 *$ \\
DBP & & $85.1 \pm 9.9$ & $79.9 \pm 10.9$ & $83.0 \pm 14.2$ & $79.2 \pm 15.5$ & $84.9 \pm 14.0$ & $49.0 \pm 7.5^{*}$ \\
HR & & $83.8 \pm 10.4$ & $78.9 \pm 14.7$ & $82.4 \pm 15.6$ & $86.4 \pm 13.5$ & $84.8 \pm 11.9$ & $52.3 \pm 5.9^{*}$ \\
\hline
\end{tabular}

* $p<0.05$ compared with $\mathrm{T}_{1}$.

TABLE 3 - Sensory nerve, motor nerve block situations in various groups $( \pm s)$.

\begin{tabular}{cccccc}
\hline Group & Case number & \multicolumn{2}{c}{ Block starting time (min) } & \multicolumn{2}{c}{ Block duration (hr) } \\
\hline & & Sensory & Motor & Sensory & Motor \\
A & 20 & $30.5 \pm 20.0$ & $120.5 \pm 29.0$ & $5.2 \pm 0.25$ & $4.0 \pm 0.22$ \\
B & 20 & $32.4 \pm 22.3$ & $125.5 \pm 32.3$ & $3.5 \pm 0.17$ & $2.0 \pm 0.15$ \\
C & 20 & $33.3 \pm 20.5$ & $0 *$ & $2.1 \pm 0.22 *$ & 0 \\
\hline
\end{tabular}

* $p<0.05$ compared with Group A. 
TABLE 4 - Ranking of the largest block degrees in various groups (Improve Bromage).

\begin{tabular}{ccccc}
\hline Group & & Intraoperative (min) & Postoperative (min) \\
\hline A & 8 & 16 & 24 & 15 \\
B & II & II & III & II \\
C & I & II & III & I \\
$0 *$ & $0 *$ & $0 *$ \\
\hline
\end{tabular}

* $p<0.05$ compared with Group A. $=$

TABLE 5 - Side effects of intraoperative and postoperative anesthetic method.

\begin{tabular}{cccccc}
\hline Group & Case number & Uroschesis (\%) & $\begin{array}{c}\text { Blood pressure } \\
\text { decreasing }(\%)\end{array}$ & Headache & Sore throat \\
\hline A & 20 & $6(30)$ & $2(10)$ & 1 & 2 \\
B & 20 & $4(20)$ & $1(5)$ & 0 & 1 \\
C & 20 & $1(5)^{*}$ & $0 *$ & 0 & 1 \\
\hline
\end{tabular}

${ }^{*} p<0.05$ compared with Group A.

\section{Discussion}

Saddle anesthesia, or caudal anesthesia, refers to numbness around the groin, across the buttocks and through the perineum, the flesh between the buttocks. When it occurs spontaneously, it can be a sign of damage to the spinal cord, and may be a symptom of a medical emergency. It can also be induced by an anesthesiologist for a medical procedure. In both cases, some neurological testing can be used to determine the extent of the loss of sensation.

The $0.5 \%$ bupivacaine has been routinely used in saddle anesthesia. In this paper, the same concentration of different doses of chloroprocaine was applied to the saddle anesthesia to investigate the feasibility and superiority of chloroprocaine in saddle anesthesia. Compared with bupivacaine, chloroprocaine can also obtain satisfactory anesthesia effect, anal relaxation, improvement of the perianal nerve block, and weak effect on indications of life (Tables 3, 4 and 5). Chloroprocaine could be used for a short acting spinal anesthetic because chloroprocaine is reliable and has a favorable safety profile to support the growing need for day case surgery. In addition, chloroprocaine is an ester type local anesthetic with the shortest duration of action of all the established local anesthetics. It has a significantly shorter duration of action than lidocaine and it is significantly less toxic than other compounds ${ }^{12}$. Based upon a retrospective review of 672 patients suitable for spinal anesthesia in surgical procedures of less than 60 minutes duration using 30-40mg chloroprocaine, chloroprocaine has a motor block lasting for 40 minutes, a rapid onset time of 3-5 minutes (9.6 min $\pm 7.3 \mathrm{~min}$ at 40 $\mathrm{mg}$ dose; $7.9 \mathrm{~min} \pm 6.0 \mathrm{~min}$ at $50 \mathrm{mg}$ dose) and a time to ambulation of 90 minutes without complications, especially without $\mathrm{TNS}^{13}$.
According to the experimental results mentioned above, the same concentration of different doses of chloroprocaine has different anesthetic effect in surgery of saddle anesthesia. The $0.5 \%(\mathrm{w} / \mathrm{v})$ chloroprocaine dissolved in 0.6 to $1.0 \mathrm{~mL} 10 \%(\mathrm{w} / \mathrm{v})$ glucose solution were employed. The patients in three groups were able to actively cooperate when changing position or transporting in intraoperative, postoperative process. Blood pressure, heart rate, oxygen saturation remained stable. In group $\mathrm{C}$, there was only sensory block of saddle area, and Bromage rating of lower limb motor block was 0-level. The hypotension after saddle anesthesia was related to the expansion of blood volume caused by spinal cord sympathetic block, and the effect of venous reflux extrusion. Blood pressure remained stable in group $\mathrm{C}$ is owing to 0-level of the lower limb motor block, lower limb tension maintain normal and normal vein blood volume. The results showed good surgical anesthesia, a fast onset time and post-operative mobilization after 90 minutes without complications.

Uroschesis is a lack of ability to urinate. It can be caused by nerve dysfunction, constipation, infection, or medications (including anticholinergics, antidepressants, COX-2 inhibitors, amphetamines and opiates). Diagnosis and/or treatment may require use of a catheter or prostatic stent. Acute uroschesis is a common complication following anorectal surgery independent of the type of anaesthesia ${ }^{14,15}$. It is linked to several risk factors such as surgical procedure, increased intravenous fluids and postoperative pain $^{16}$. Both the innervation in saddle area and urinary system belong to the same nerve segment (S2-S4), leading to uroschesis in postoperative, requiring catheterization. There is one case of uroschesis in group $\mathrm{C}$, which may be associated with wound pain, hemorrhage, edema and gauze fill pressure. 
Taking into account all aspects of group A, group B and group $\mathrm{C}$ with the same concentration $(0.5 \%)$ chloroprocaine with different doses $1.0 \mathrm{~mL}, 0.8 \mathrm{~mL}$ and $0.6 \mathrm{~mL}$, respectively. Group C is superior to other two groups in terms of analgesic consumption within 24 hours after surgery and aspects of postoperative recovery.

\section{Conclusion}

It's worth to employ a saddle anesthesia with appropriate doses of chloroprocaine in clinical perianal surgery.

\section{References}

1. Brown MD, Levi AD. Surgery for lumbar disc herniation during pregnancy. Spine. 2001;26(4):440-3.

2. Schmittner M, Schreiber H, Janke A, Weiss C, Blunk J, Bussen D, Luecke T. Randomized clinical trial of perianal surgery performed under spinal saddle block versus total intravenous anaesthesia. Br J Surg. 2010;97(1):12-20.

3. Chaudhari L, Kane D, Shivkumar B, Kamath S. Comparative study of intrathecal pethidine versus lignocaine as an anaesthetic and a postoperative analgesic for perianal surgery. J Postgrad Med. 1996;42(2):43-5.

4. Abboud TK, Khoo SS, Miller F, Doan T, Henriksen EH. Maternal, fetal, and neonatal responses after epidural anesthesia with bupivacaine, 2-chloroprocaine, or lidocaine. Anesth Analg. 1982;61(8):638-44.

5. Kuhnert B, Kuhnert P, Prochaska A, Gross T. Plasma levels of 2-chloroprocaine in obstetric patients and their neonates after epidural anesthesia. Anesthesiology. 1980;53(1):21-5.

6. Stevens RA, Urmey WF, Urquhart BL, Kao T-C. Back pain after epidural anesthesia with chloroprocaine. Anesthesiology. 1993;78(3):492-7.

7. Abboud TK, DerSarkissian L, Terrasi J, Murakawa K, Zhu J, Longhitano M. Comparative maternal, fetal, and neonatal effects of chloroprocaine with and without epinephrine for epidural anesthesia in obstetrics. Anesth Analg. 1987;66(1):71-5.

8. Marica LS, O'Day T, Janosky JE, Nystrom EU. Chloroprocaine is less painful than lidocaine for skin infiltration anesthesia. Anesth Analg. 2002;94(2):351-4.

9. Turkmen A, Moralar DG, Ali A, Altan A. Comparison of the anesthetic effects of intrathecal levobupivacaine + fentanyl and bupivacaine + fentanyl during caesarean section. Middle East J Anesthesiol 2012;21:577-82.
10. Hodgkinson R, Bhatt M, Wang CN. Double-blind comparison of the neurobehaviour of neonates following the administration of different doses of meperidine to the mother. Can Anaesth Soc J. 1978;25(5):405-11.

11. Bromage PR, Benumof JL. Paraplegia following intracord injection during attempted epidural anesthesia under general anesthesia. Reg Anesth Pain Med. 1998;23(1):104-7.

12. Goldblum E, Atchabahian A.The use of 2-chloroprocaine for spinal anaesthesia. Acta Anaesthesiol Scand 2013;57:545-52.

13. Campiglio GL, Bufo S, De Sisto L, Garassino E, Gibelli PL, Gilardino P, Lucchesi R, Muti GF, Orlandi A, Rapisarda V, Tesauro P, Todde A; Associazione Lombarda di Chirurgia Plastica Ambulatoriale. [guidelines for the safe outpatient practice of aesthetic surgery]. Minerva Chir 2010;65:145-52.

14. Salvati EP, Kleckner MS. Urinary retention in anorectal and colonic surgery. Am J Surg. 1957;94(1):114-7.

15. Scoma JA. Catheterization in anorectal surgery. Arch Surg. 1975;110(12):1506.

16. Toyonaga T, Matsushima M, Sogawa N, Jiang SF, Matsumura N, Shimojima Y, Tanaka Y, Suzuki K, Masuda J, Tanaka M. Postoperative urinary retention after surgery for benign anorectal disease: potential risk factors and strategy for prevention. Int $\mathrm{J}$ Colorectal Dis. 2006;21(7):676-82.

\section{Correspondence:}

Dongping Shi

Department of Anesthesiology, Shanghai Jiading Central Hospital $N^{o} 1$ Chengbei Road, Jiading District

Shanghai 201800, China.

Phone: +86-021-67073174

Fax: +86-021-67073174

dongpingshisdp@hotmail.com

Received: Sept 17, 2013

Review: Nov 18, 2013

Accepted: Dec 20, 2013

Conflict of interest: none

Financial source: Youth Foundation of Jiading District (Grant $\mathrm{n}^{\circ}$ 2013QN-01)

${ }^{1}$ Research performed at Department of Anesthesiology, Shanghai Jiading Central Hospital, Shanghai, China. 